\title{
Trends in surgical volume and in-hospital mortality among United States cirrhosis hospitalizations
}

\author{
Kristen M. Tessiatore ${ }^{a}$, Nadim Mahmud ${ }^{b, c}$ \\ University of Pennsylvania, Philadelphia, PA, USA
}

\section{Abstract}

${ }^{a}$ Department of Internal Medicine, Perelman School of Medicine (Kristen M. Tessiatore); ' Division of Gastroenterology, Perelman School of Medicine (Nadim Mahmud); ' Leonard David Institute of Health Economics (Nadim Mahmud), University of Pennsylvania, Philadelphia, PA, USA

Conflict of Interest: None

Correspondence to: Nadim Mahmud, MD, MS, MPH, MSCE, Division of Gastroenterology, University of Pennsylvania, 3400 Civic Center Blvd, Philadelphia, PA 19104, USA,

E-mail: mahmudn@pennmedicine.upenn.edu

Received 11 June 2020; accepted 4 August 2020; published online 20 November 2020

DOI: https://doi.org/10.20524/aog.2020.0554

\section{Introduction}

The overall prevalence of cirrhosis in the United States (US) is estimated to be $0.27 \%$ of the general US population, or greater than 600,000 individuals [1]. A recent national study demonstrated a $25 \%$ increase in the number of hospitalizations of patients with cirrhosis from 2005-2014, with the majority of these patients being aged 51-69 years [2]. This reflects an aging population of patients with cirrhosis, who will presumably require higher rates of surgical procedures.

The data on national trends in surgical procedure volume and mortality among patients with cirrhosis are sparse. A study of national discharge data from 1998-2005 found stable surgical volumes among cirrhosis admissions, with 
cholecystectomies performed much more commonly than major cardiovascular surgery, in contrast to non-cirrhosis admissions [3]. However, there have been no US national data published on cirrhosis surgical volume since that time. We recently demonstrated significant differences in in-hospital mortality by surgery type among cirrhosis admissions in the National Inpatient Sample database [4]. However, no recent studies have evaluated trends in volumes of major surgery in this population, which could serve to further contextualize these findings. In order to address these gaps, we sought to quantify temporal trends in surgical procedures among admissions with cirrhosis, as well as in-hospital mortality rates by surgical procedure type, elective or non-elective admission status, and by decompensation status.

\section{Materials and Methods}

We performed a retrospective cohort study using complete data from the Nationwide Inpatient Sample (2005-2011) and National Inpatient Sample (2012-2014), collectively referred to here as the NIS. The NIS is the largest payer-independent database of US hospital discharges, and is maintained by the Agency for Healthcare Research and Quality (AHRQ) as part of the Healthcare Cost Utilization Project (HCUP) [5].

We combined the NIS datasets from 2005-2014, and identified all admission events with age $\geq 18$ and an International Classification of Diseases (ICD)-9 diagnosis code for cirrhosis $(571.2,571.5)$. We classified admissions with decompensated cirrhosis using codes for variceal bleed (456.0, 456.2), hepatic encephalopathy (572.2), portal hypertension (572.3), hepatorenal syndrome (572.4) or ascites (789.5), consistent with prior NIS studies [3,6]. Demographic data (age, sex, race), primary insurance payer (Medicare, Medicaid, private, self-pay), hospital length of stay, hospital location/ teaching status (rural, urban nonteaching, urban teaching), hospital region (Northeast, Midwest, South, West), hospital size (small, medium, large) based on number of beds [7], elective vs. non-elective admission, and in-hospital death were collected for all admissions. Elixhauser comorbidity indices were also calculated for each admission [8]. This is a validated index for hospital-associated mortality that outperforms other comorbidity indices across a range of applications utilizing large administrative databases [9]. Finally, we classified the etiology of liver disease as hepatitis $\mathrm{C}$, alcohol-related, fatty liver disease or unclassified/missing, based on prior methods [4].

We reviewed the 100 most common primary procedure codes from the annual data of all admissions in the cohort and created 5 surgical subcategories: cholecystectomy, hernia repair, major abdominal (including total/partial colectomy, nephrectomy, pancreatectomy, splenectomy, gastrectomy) [10], major orthopedic (including total/partial knee or hip replacement/revision, major open reduction procedure) [11], and major cardiovascular (including coronary artery bypass grafting, open cardiac valve replacement/repair, abdominal aortic aneurysm repair). ICD-9 Clinical Modification procedure codes are summarized in Supplementary Table 1.
We used NIS discharge weights, including adjusted trend weights for pre-2012 data, to produce national estimates of admissions with surgical procedures of interest [5]. Descriptive statistics were presented as medians and interquartile ranges for continuous variables and as percentages for categorical variables. Aggregate in-hospital mortality rates for each surgical procedure were calculated by dividing the number of hospitalizations ending in death by the total number of hospitalizations with a given surgical procedure. In order to assess trends, we tabulated and plotted the number of surgical procedures over time, stratified by procedure category. As trend lines appeared linear, we used univariable linear regression to estimate beta coefficients and test against a null hypothesis of beta $=0$, using a P-value threshold of $<0.05$ for statistical significance. To determine trends in surgical volume accounting for rising numbers of cirrhosis admissions, we repeated the above approach with all estimates divided by the number of national cirrhosis admissions (per 1000 admissions). In exploratory analyses, we produced plots of overall surgical volume and associated in-hospital mortality over time as stratified based on elective or non-elective admission status, as well as by compensated or decompensated cirrhosis status. For comparisons of beta coefficients between trend lines, we performed the Chow test.

\section{Results}

There were 221,296 surgical procedures of interest performed on patients admitted with cirrhosis from 2005-2014 (Table 1). Major abdominal and major cardiovascular surgeries had the highest elective admission rates $(51.1 \%$ and $51.2 \%$, respectively) and the highest median length of stay (9 and 10 days, respectively). Patients who underwent hernia repair or major orthopedic surgery were most likely to have alcoholrelated liver disease ( $36.3 \%$ and $36.8 \%$, respectively). The Elixhauser comorbidity index was highest in those undergoing major cardiovascular surgeries. In-hospital death rates were highest among those undergoing major abdominal (14.7\%) and cardiovascular surgeries (9.9\%), and lowest among those undergoing major orthopedic procedures (2.2\%).

The estimated number of hospital admissions with cirrhosis increased significantly over time, from 380,246 in 2005 to 604,385 in 2014 ( $\beta=25,639, \mathrm{P}<0.001$; Table 2 ). Similarly, the absolute number of surgical procedures increased from 20,717 to 25,405 over the same period $(\beta=499.05, \mathrm{P}<0.001)$. While the most commonly performed procedure in each year was cholecystectomy, the highest rate of increase in overall surgical volume was observed in major orthopedic procedures, followed by hernia repairs, and then major cardiovascular surgeries $(\beta=310.77, P<0.001 ; \beta=159.32$, $\mathrm{P}<0.001 ; \beta=55.99, \mathrm{P}=0.026$; Fig. $1 \mathrm{~A}$, Table 2).

While the number of hospital admissions with cirrhosis increased over time, the number of surgeries per 1,000 cirrhosis admissions decreased from 2005 to $2014(\beta=-1.454, \mathrm{P}<0.001$; Supplementary Table 2, Fig. 1B). The rates of cholecystectomy declined the most precipitously (22.3 per 1,000 in 2005 vs. 12.7 per 1,000 in $2014 ; \beta=-1.004, \mathrm{P}<0.001$ ), followed by 
Table 1 Hospitalization characteristics for 2004-2014 cohort

\begin{tabular}{|c|c|c|c|c|c|}
\hline Variable & $\begin{array}{l}\text { Cholecystectomy } \\
\qquad(\mathrm{N}=76,950)\end{array}$ & $\begin{array}{l}\text { Hernia repair } \\
(\mathrm{N}=44,878)\end{array}$ & $\begin{array}{l}\text { Other major abdominal } \\
\qquad(\mathrm{N}=34,158)\end{array}$ & $\begin{array}{l}\text { Major orthopedic } \\
\quad(\mathrm{N}=50,638)\end{array}$ & $\begin{array}{l}\text { Major cardiovascular } \\
\qquad(\mathrm{N}=14,672)\end{array}$ \\
\hline Age (years; median, IQR) & $60(52-71)$ & $58(52-66)$ & $61(54-71)$ & $62(55-71)$ & $64(56-71)$ \\
\hline Female (\%) & 43.7 & 31.3 & 41.8 & 50.2 & 26.0 \\
\hline \multicolumn{6}{|l|}{ Race (\%) } \\
\hline White & 66.9 & 70.6 & 72.3 & 78.7 & 76.0 \\
\hline Black & 8.8 & 8.6 & 8.5 & 6.5 & 6.8 \\
\hline Hispanic & 17.0 & 16.1 & 13.2 & 10.2 & 11.0 \\
\hline \multicolumn{6}{|l|}{ Primary insurance (\%) } \\
\hline Medicare & 46.4 & 41.6 & 46.9 & 56.5 & 54.1 \\
\hline Medicaid & 14.4 & 19.6 & 13.5 & 13.6 & 12.9 \\
\hline Private & 28.1 & 28.2 & 29.7 & 23.3 & 25.3 \\
\hline Self-pay & 6.3 & 5.4 & 5.7 & 3.0 & 3.9 \\
\hline $\begin{array}{l}\text { Elixhauser comorbidity index } \\
\text { (median, IQR) }\end{array}$ & $4(2-5)$ & $4(3-5)$ & $5(3-6)$ & $4(2-5)$ & $6(5-8)$ \\
\hline \multicolumn{6}{|l|}{ Etiology of liver disease (\%) } \\
\hline Hepatitis C & 13.1 & 14.9 & 9.3 & 11.1 & 11.5 \\
\hline Alcohol & 20.9 & 36.3 & 27.4 & 36.8 & 31.7 \\
\hline Fatty liver & 10.9 & 7.7 & 12.6 & 7.3 & 8.4 \\
\hline Unclassified/missing & 55.1 & 41.1 & 50.7 & 44.8 & 48.4 \\
\hline Decompensated cirrhosis (\%) & 19.7 & 37.4 & 25.7 & 17.8 & 17.9 \\
\hline $\begin{array}{l}\text { Length of stay (days; median, } \\
\text { IQR) }\end{array}$ & $6(3-9)$ & $5(3-10)$ & $9(5-16)$ & $5(3-8)$ & $10(6-17)$ \\
\hline Elective admission (\%) & 29.5 & 33.2 & 51.1 & 41.8 & 51.2 \\
\hline \multicolumn{6}{|l|}{ Region (\%) } \\
\hline Northeast & 14.4 & 17.1 & 17.3 & 17.7 & 18.5 \\
\hline Midwest & 18.2 & 20.0 & 20.5 & 20.4 & 20.1 \\
\hline South & 41.3 & 37.6 & 39.3 & 39.2 & 40.1 \\
\hline West & 26.1 & 25.3 & 22.9 & 22.8 & 21.3 \\
\hline \multicolumn{6}{|l|}{ Hospital size } \\
\hline Small & 10.9 & 10.1 & 10.1 & 12.5 & 5.5 \\
\hline Medium & 24.5 & 23.9 & 22.9 & 25.4 & 18.6 \\
\hline Large & 64.6 & 66.0 & 66.8 & 62.0 & 75.9 \\
\hline \multicolumn{6}{|c|}{ Location/teaching status of hospital } \\
\hline Rural & 11.6 & 7.7 & 7.9 & 9.3 & 2.5 \\
\hline Urban nonteaching & 41.8 & 35.5 & 34.5 & 41.9 & 28.5 \\
\hline Urban teaching & 46.6 & 56.8 & 57.5 & 48.8 & 69.0 \\
\hline Inpatient death (\%) & 3.5 & 4.2 & 14.7 & 2.2 & 9.9 \\
\hline $\begin{array}{l}\text { Hospital charges } \\
\text { (\$; median, IQR) }\end{array}$ & $\begin{array}{c}48,318 \\
(28,045-92,271)\end{array}$ & $\begin{array}{c}45,049 \\
(24,897-93,337)\end{array}$ & $\begin{array}{c}80,639 \\
(45,651-152,326)\end{array}$ & $\begin{array}{c}52,031 \\
(35,013-82,672)\end{array}$ & $\begin{array}{c}150,547 \\
(94,492-258,331)\end{array}$ \\
\hline
\end{tabular}

$I Q R$, interquartile range

major abdominal surgery ( 8.9 vs. $6.3 ; \beta=-0.316 ; \mathrm{P}<0.001)$ and hernia repair ( 8.9 vs. $6.3 ; \beta=-0.316 ; \mathrm{P}<0.001$ ). The only surgical subcategory volume to increase over time was major orthopedic surgeries, though this change was not statistically significant (from 9.9 to 11.4 per 1,000 cirrhosis admissions; $\beta=0.083, P=0.078$ ).

When surgical procedure volume was stratified by elective admission status (Fig. 2A), elective major orthopedic 
Table 2 National estimates and trends in hospital surgical procedures among cirrhosis hospitalizations

\begin{tabular}{lccccccc}
\hline $\begin{array}{l}\text { Discharge } \\
\text { year }\end{array}$ & $\begin{array}{c}\text { Cholecystectomy } \\
(\mathrm{N}=76,950)\end{array}$ & $\begin{array}{c}\text { Hernia repair } \\
(\mathrm{N}=44,878)\end{array}$ & $\begin{array}{c}\text { Other major } \\
\text { Abdominal } \\
(\mathrm{N}=34,158)\end{array}$ & $\begin{array}{c}\text { Major } \\
\text { orthopedic } \\
(\mathrm{N}=50,638)\end{array}$ & $\begin{array}{c}\text { Major } \\
\text { cardiovascular } \\
(\mathrm{N}=14,672)\end{array}$ & $\begin{array}{c}\text { Total surgeries } \\
(\mathrm{N}=221,296)\end{array}$ & $\begin{array}{c}\text { Total } \\
\text { cirrhosis admissions } \\
(\mathrm{N}=4,824,116)\end{array}$ \\
\hline 2005 & 8394 & 3900 & 3383 & 3776 & 1265 & 20717 & 380246 \\
2006 & 7812 & 3874 & 3596 & 4079 & 1458 & 20820 & 404908 \\
\hline 2007 & 7526 & 4165 & 3270 & 4316 & 1257 & 20534 & 414285 \\
\hline 2008 & 7803 & 4109 & 2989 & 4607 & 1200 & 20708 & 421855 \\
\hline 2009 & 7328 & 4212 & 3314 & 4860 & 1665 & 21379 & 457917 \\
\hline 2010 & 7860 & 4692 & 3326 & 4932 & 1223 & 22033 & 484560 \\
\hline 2011 & 7841 & 4736 & 3434 & 5369 & 1509 & 22890 & 532159 \\
\hline 2012 & 7640 & 4805 & 3430 & 5800 & 1480 & 23155 & 552525 \\
\hline 2013 & 7080 & 5260 & 3625 & 6035 & 1655 & 23655 & 571275 \\
\hline 2014 & 7665 & 5125 & 3790 & 6865 & 1960 & 25405 & 604385 \\
\hline $\begin{array}{l}\text { Linear trend, } \\
(\beta, \mathrm{P} \text {-value })\end{array}$ & -63.46 & $159.32^{*}$ & 36.43 & $310.77^{*}$ & $55.99^{*}$ & $499.05^{*}$ & $25639.80^{*}$ \\
\hline${ }^{*}<0.05$ & $(\mathrm{P}=0.101)$ & $(\mathrm{P}<0.001)$ & $(\mathrm{P}=0.142)$ & $(\mathrm{P}<0.001)$ & $(\mathrm{P}=0.026)$ & $(\mathrm{P}<0.001)$ & $(\mathrm{P}<0.001)$ \\
\hline
\end{tabular}

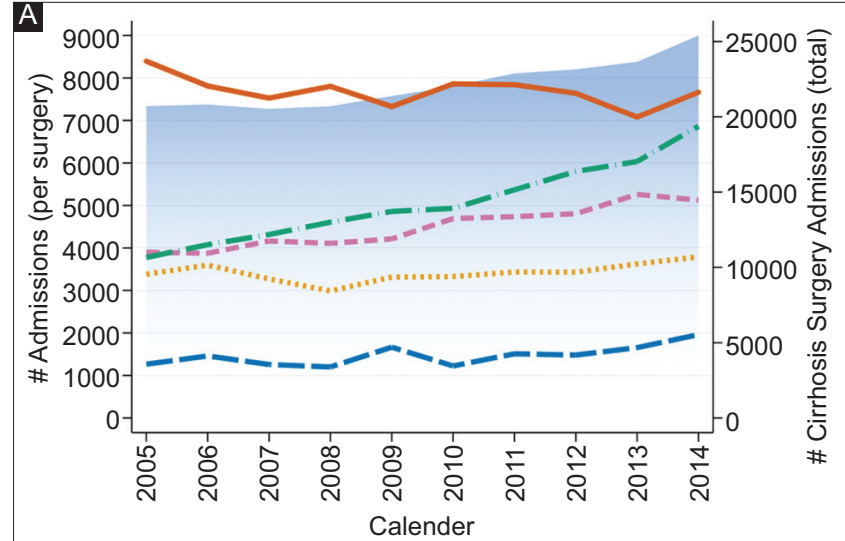

- Cholecystectomy $=-$ Hernia repair.... Major abdominal - Major orthopedic — Major cardiovascular Total

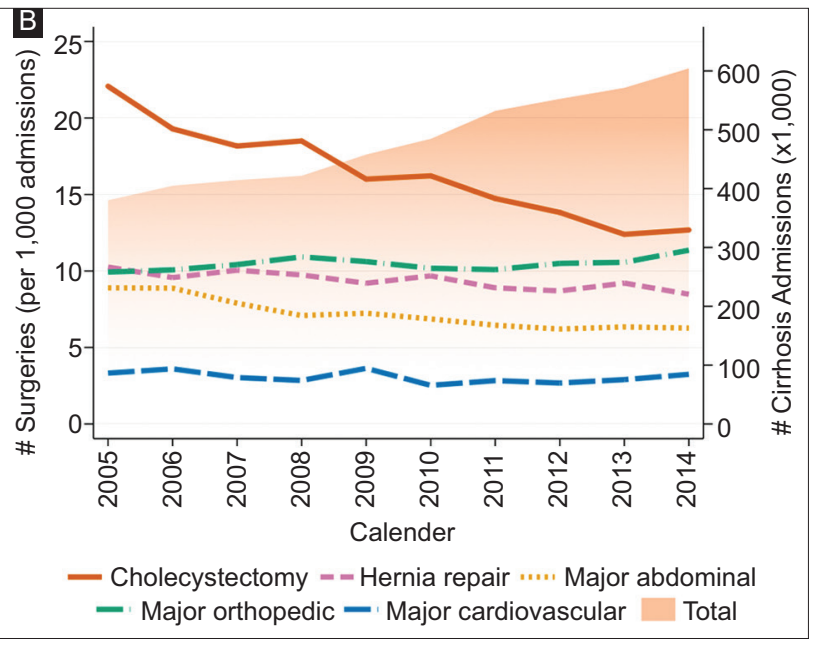

Figure 1 (A, B) National trends in cirrhosis surgery admissions by overall volume (A) and per 1000 cirrhosis admissions (B)

surgeries increased significantly over time $(\beta=177.9 ; \mathrm{P}<0.001)$, surpassing cholecystectomy as the most common procedure by 2011. Cholecystectomy, hernia repairs and major abdominal surgeries were performed significantly more often in the non-elective than in the elective setting (each $\mathrm{P}<0.001$ ), with cholecystectomy being the most common non-elective procedure overall. Major orthopedic procedures and hernia repairs had significantly increasing annual volumes among non-elective admissions $(\beta=131.4 ; \mathrm{P}<0.001$ and $\beta=134.0$; $\mathrm{P}<0.001)$.

In-hospital mortality rates for major abdominal, major cardiovascular, hernia repair and major orthopedic surgeries were significantly higher in the non-elective vs. elective setting (each $\mathrm{P}<0.001$, Fig. 2B). For elective admissions, in-hospital mortality rates were $\leq 10 \%$ over the entire study period. Major abdominal surgeries had the overall highest in-hospital mortality rate among non-elective admissions, exceeding $20 \%$ across all time points. Mortality rates of major abdominal surgeries declined significantly in the elective setting $(\beta=-0.005$; $\mathrm{P}=0.013)$ but not in the non-elective setting $(\beta=-0.00 ; \mathrm{P}=0.275)$. Major orthopedic surgeries generally maintained the lowest inpatient mortality rate amongst both groups.

When cirrhosis surgery admissions were stratified by compensated or decompensated status, we observed significant increases in the absolute number of major orthopedic $(\beta=272.4 ; \mathrm{P}<0.001)$ and hernia repair $(\beta=191.1 ; \mathrm{P}<0.001)$ surgeries over time among patients with compensated cirrhosis (Fig. 3A). Trends in surgery volume were generally stable over time among patients with decompensated cirrhosis, and were numerically lower for each category as compared to patients with compensated cirrhosis. Regarding in-hospital mortality, decompensated cirrhosis conferred universally higher inhospital mortality relative to compensated cirrhosis for each surgery type (Fig. 3B; each $\mathrm{P}<0.05$ Q. Please mention the 


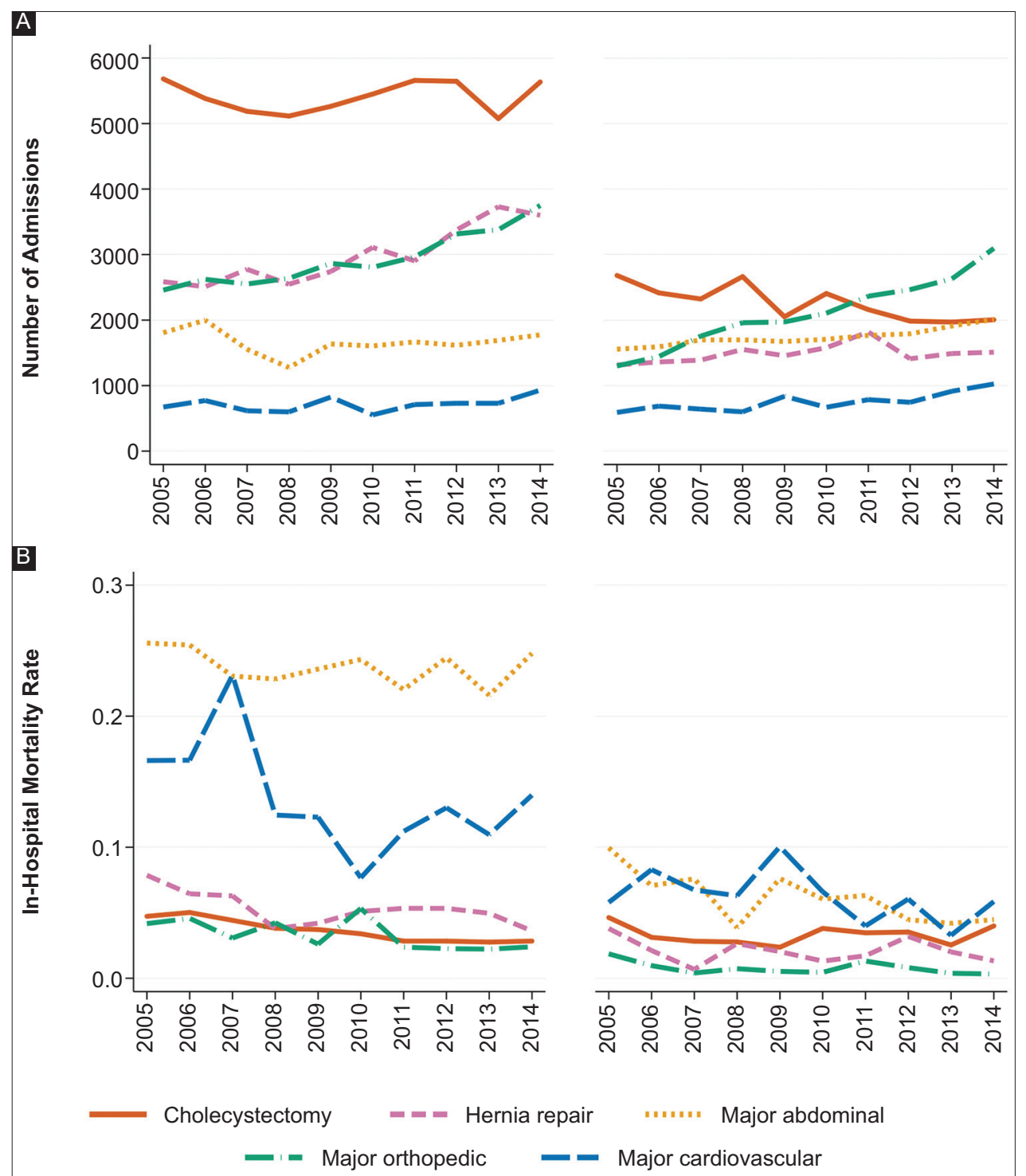

Figure 2 National trends in cirrhosis surgery admissions (A) and associated in-hospital mortality rates (B), each stratified by elective or nonelective admission status

exact P-value unless $<0.001)$. In particular, although there was a trend towards decreasing in-hospital mortality for major abdominal surgery among patients with decompensated cirrhosis $(\beta=-0.006, \mathrm{P}=0.069)$, the in-hospital mortality rate remained significantly greater than that observed for patients with compensated cirrhosis (8.1\% higher in 2014, $\mathrm{P}<0.001)$.

\section{Discussion}

In this large national study of data spanning more than one decade, we identified common surgical procedure categories for cirrhosis hospitalizations and revealed changes in overall national trends. We additionally performed analyses addressing differences by elective admission status, compensated vs. decompensated status, as well as trends in inhospital mortality.

First, our study demonstrated that while the number of cirrhosis admissions and absolute surgical volume is increasing over time, the number of surgeries per 1,000 cirrhosis admissions is decreasing. There are several possible explanations for this finding. First, as the population of patients with cirrhosis is aging, a similar need for surgery may result in excess hospitalization and hospital readmission for post-surgical complications. Second, the data may alternatively suggest an increasingly conservative approach to surgery that may potentially be explained by risk aversion attributable to a higher-risk patient population. A statewide population-based study found that new diagnoses of cirrhosis increased by $69 \%$ (from 620 to 1045) between 2004 and 2014 [12], with the greatest increase occurring in patients 


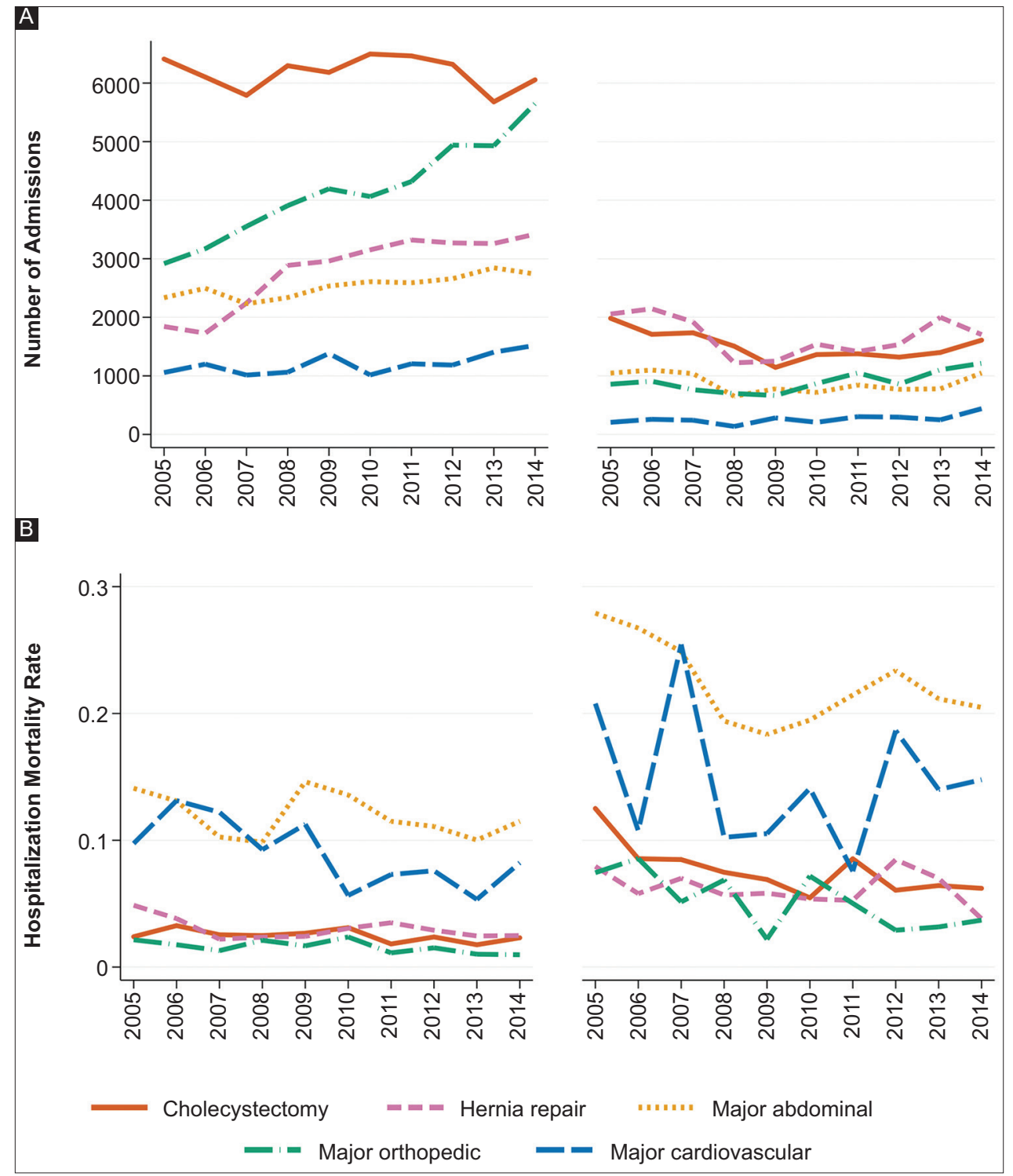

Figure 3 National trends in cirrhosis surgery admissions (A) and associated in-hospital mortality rates (B), each stratified by compensated or decompensated cirrhosis status

aged 65 years and older. Over the same time period, a study of 126 Veteran Administration hospitals showed that, while in-hospital mortality decreased from $11.4 \%$ to $7.6 \%$ among cirrhosis hospitalizations, 30-day post-discharge mortality increased from $9.3 \%$ to $10.1 \%$ [13]. Taken together, these data raise the question of whether an aging and frailer population of patients with cirrhosis could account for the relative decline in rates of invasive surgical procedures being offered to these individuals. Third, an additional explanation for risk aversion could be the expanded use of preoperative cirrhosis surgical risk scores such as the Mayo risk score [14], which has been demonstrated to overestimate surgical risk [15]. This is important, as risk aversion is apparently progressing despite relatively stable or potentially reduced in-hospital mortality among certain groups of patients (e.g., elective major abdominal surgery). This implies that preoperative risk-stratification tools may need to be reassessed and/or recalibrated for the modern surgical era, as has been argued recently [16]. Future research is needed to distinguish between the above hypotheses.

Second, we found an increasing volume of major orthopedic surgeries among patients with cirrhosis, regardless of elective admission status, although the rates per 1000 cirrhosis admissions have remained generally stable over time. Other studies have demonstrated that the volume of both total knee (TKR) and total hip (THR) replacements has been increasing annually among the general population. Cram et al reported a 161.5\% overall increase in TKR from 1991-2010 in a Medicare population [17]. Nwachukwu et al found a $1.5 \%$ annual increase in THR from 2005-2011 [18]. These trends probably reflect the demonstrated improvements in quality of life and mobility in concert with the acceptable safety profile 
of these procedures $[19,20]$. In our study, the increase in major orthopedic procedures among elective cirrhosis admissions was more than 200\% from 2005-2014. This notable trend may correspond to the expanding awareness that frailty and poor functional status increase mortality for patients with cirrhosis, in addition to the lower perceived risk of these procedures [21,22]. As such, elective procedures to improve mobility and decrease pain may be beneficial in selected patients.

Third, in an exploratory analysis of surgery-specific mortality trends, we first noted significantly higher inhospital mortality rates when procedures were performed in the non-elective setting. The notable exception, however, was in the case of cholecystectomy, where in-hospital mortality rates were similar for elective and non-elective hospitalizations. This is a surprising finding, as the prevailing literature suggests a uniformly higher mortality rate with cholecystectomies performed under emergent circumstances. Our analysis also showed wide variation in in-hospital mortality rates by procedure type, primarily among nonelective cases. This is consistent with recently published literature demonstrating differences in short-term mortality by surgery type in adjusted models $[4,23]$. In particular, we found extremely high in-hospital mortality among patients undergoing non-elective major abdominal surgery, and among decompensated patients undergoing major abdominal surgery. The grave prognosis in these circumstances should be incorporated into clinical practice when considering surgical management, and in prognostic discussions with patients and healthcare proxies. Stated more broadly, the data presented herein suggest that future efforts to risk stratify patients with cirrhosis preoperatively should take account of the surgical procedure type, as well as the elective or non-elective surgical indication.

Finally, in a subgroup analysis of surgery admissions and in-hospital mortality stratified by compensated vs. decompensated cirrhosis status, we noted a significant increase in the absolute number of major orthopedic surgeries over time among patients with compensated cirrhosis. The current literature suggests higher rates of complications and mortality, and a longer length of hospital stay for orthopedic procedures compared with non-cirrhotic patients; however, these studies to do not stratify by compensated or decompensated status [24,25]. We also noted an increase in the total volume of hernia repair surgeries among patients with compensated cirrhosis over time, in line with current findings in the literature. In a recent retrospective review the mortality rate in patients with cirrhosis undergoing umbilical hernia repair was $1.3 \%$ for a model for end-stage liver disease (MELD) score $<15$ and $11.1 \%$ in patients with MELD 15 or greater [26]. Cholecystectomy remained the most common procedure performed over time in the compensated group. This can be attributed to the low complication rates and reduced hospital length of stay associated with laparoscopic cholecystectomy in patients with cirrhosis $[27,28]$. We also noted significantly higher rates of in-hospital mortality among patients with decompensated cirrhosis as compared to patients with compensated cirrhosis undergoing surgical procedures. These findings are consistent with the established literature that patients with decompensated cirrhosis are at higher risk of infection, bleeding, hepatic encephalopathy, poor nutritional status, and hemodynamic, renal and cardiopulmonary complications compared to individuals with compensated cirrhosis. In a 2006 systematic review of 118 studies, median survival time in patients with compensated vs. decompensated cirrhosis was greater than 12 years and around 2 years, respectively [29]. A recently published review of 87 studies found that, generally for elective procedures, patients with compensated cirrhosis had a minimal increase in perioperative mortality and that patients with decompensated cirrhosis had higher rates of mortality [30]. Therefore, compensated vs. decompensated status should be factored into preoperative risk assessment when evaluating patients with cirrhosis for surgery.

There are several limitations that we acknowledge in this study. First, as with any administrative database study, there is possible misclassification due to coding errors. This could cause over- or underestimation of observed trends; however, in aggregate we would expect this impact to be minimal [4]. Second, the use of in-hospital mortality as a metric does not capture patients who die shortly after discharge. This limitation of the NIS dataset warrants caution in interpreting the findings of this study with regard to short-term mortality after surgery in patients with cirrhosis. Third, by the nature of the NIS database, this analysis does not incorporate outpatient surgeries that do not involve hospitalization, expected to have a different risk profile. This may impact the external validity of this study, and it is important to highlight that the observed data reflect major surgical procedures requiring hospitalization. Fourth, the NIS database does not include laboratory data that would allow us to evaluate the impact of MELD score on surgical risk. It is additionally challenging to classify chronic comorbidities not directly related to hospitalization, as administrative codes are only supplied for individual admissions. Finally, we intentionally did not compare surgical trends or outcomes to patients without cirrhosis, as it is well-established that cirrhosis confers a greater surgical risk relative to patients without cirrhosis. Furthermore, the goal of this work was to focus on salient differences in surgical procedure types among patients with cirrhosis.

In conclusion, in this large analysis of national hospitalization data, we identified notable changes in surgical procedure trends among cirrhosis hospitalizations between 2005 and 2014. In-hospital mortality was higher among patients admitted non-electively, and varied substantially based on the type of surgery performed. Additional studies are needed to confirm and elucidate the underlying mechanism of these findings. However, our study highlights the need for risk prediction models that take account of surgical procedure type, elective/non-elective surgical indication, and decompensation status. 


\section{Summary Box}

\section{What is already known:}

- The number of cirrhosis hospitalizations in the United States is rising

- The population of patients with cirrhosis is aging, and the need for surgeries may also be increasing

- There are limited data investigating changing cirrhosis surgical trends at the national level

\section{What the new findings are:}

- Although the number of cirrhosis hospitalizations is increasing, the number of surgeries per 1000 admissions is decreasing; this may reflect a culture of risk aversion in cirrhosis surgery

- Elective major orthopedic surgeries have been increasing over time

- In-hospital mortality is higher among surgeries performed non-electively or among patients with decompensated cirrhosis

\section{References}

1. Scaglione S, Kliethermes S, Cao G, et al. The epidemiology of cirrhosis in the United States: a population-based study. J Clin Gastroenterol 2015;49:690-696.

2. Peery AF, Crockett SD, Murphy CC, et al. Burden and cost of gastrointestinal, liver and pancreatic diseases in the United States; update 2018. Gastroenterology 2019;156:254-272.e11.

3. Csikesz NG, Nguyen LN, Tseng JF, Shah SA. Nationwide volume and mortality after elective surgery in cirrhotic patients. J Am Coll Surg 2009;208:96-103.

4. Mahmud N, Fricker Z, Serper M et al. In-hospital mortality varies by procedure type among cirrhosis admissions. Liver Int 2019;39:1394-1399.

5. Healthcare Cost Utilization Project: NIS database documentation. Volume 2019. Rockville, MD: Agency for Healthcare Research and Quality, 2018.

6. Sam J, Nguyen GC. Protein-calorie malnutrition as a prognostic indicator of mortality among patients hospitalized with cirrhosis and portal hypertension. Liver Int 2009;29:1396-1402.

7. NIS Description of Data Elements. Healthcare Cost and Utilization Project (HCUP). Volume 2019. Rockville, MD: Agency for Healthcare Research and Quality, 2018.

8. Elixhauser A, Steiner C, Harris DR, Coffey RM. Comorbidity measures for use with administrative data. Med Care 1998;36:8-27.

9. Menendez ME, Neuhaus V, van Dijk CN, Ring D. The Elixhauser comorbidity method outperforms the Charlson index in predicting inpatient death after orthopaedic surgery. Clin Orthop Relat Res 2014;472:2878-2886.

10. Mukherjee D, Lidor AO, Chu KM, Gearhart SL, Haut ER, Chang DC. Postoperative venous thromboembolism rates vary significantly after different types of major abdominal operations. $J$ Gastrointest Surg 2008;12:2015-2022.

11. Taylor HD, Dennis DA, Crane HS. Relationship between mortality rates and hospital patient volume for Medicare patients undergoing major orthopaedic surgery of the hip, knee, spine, and femur. $J$ Arthroplasty 1997;12:235-242.

12. Orman ES, Roberts A, Ghabril M, et al. Trends in characteristics, mortality, and other outcomes of patients with newly diagnosed cirrhosis. JAMA Netw Open 2019;2:e196412.

13. Kanwal F, Tansel A, Kramer JR, Feng H, Asch SM, El-Serag HB. Trends in 30-day and 1-year mortality among patients hospitalized with cirrhosis from 2004 to 2013.Am J Gastroenterol 2017;112:12871297.

14. Teh SH, Nagorney DM, Stevens SR, et al. Risk factors for mortality after surgery in patients with cirrhosis. Gastroenterology 2007;132:1261-1269.

15. Kim SY, Yim HJ, Park SM, et al. Validation of a Mayo post-operative mortality risk prediction model in Korean cirrhotic patients. Liver Int 2011;31:222-228.

16. Wong NZ, Mahmud N. The imperative for an updated cirrhosis surgical risk score. Ann Hepatol 2020;19:341-343.

17. Cram P, Lu X, Kates SL, Singh JA, Li Y, Wolf BR. Total knee arthroplasty volume, utilization, and outcomes among Medicare beneficiaries, 1991-2010. JAMA 2012;308:1227-1236.

18. Nwachukwu BU, McCormick F, Provencher MT, Roche M, Rubash HE. A comprehensive analysis of Medicare trends in utilization and hospital economics for total knee and hip arthroplasty from 2005 to 2011. J Arthroplasty 2015;30:15-18.

19. Manley M, Ong K, Lau E, Kurtz SM. Total knee arthroplasty survivorship in the United States Medicare population: effect of hospital and surgeon procedure volume. J Arthroplasty 2009;24:1061-1067.

20. Cram P, Vaughan-Sarrazin MS, Wolf B, et al. A comparison of total hip and knee replacement in specialty and general hospitals. J Bone Joint Surg Am 2007;89:1675-1684.

21. Tapper EB, Finkelstein D, Mittleman MA, Piatkowski G, Lai M. Standard assessments of frailty are validated predictors of mortality in hospitalized patients with cirrhosis. Hepatology 2015;62:584590.

22. Lai JC, Feng S, Terrault NA, Lizaola B, Hayssen H, Covinsky K. Frailty predicts waitlist mortality in liver transplant candidates. Am J Transplant 2014;14:1870-1879.

23. Fricker Z, Mahmud N, Goldberg DS, et al. An improved model to predict cirrhosis surgical risk. Hepatology 2019;70 (Suppl 1):36A.

24. Deleuran T, Vilstrup H, Overgaard S, Jepsen P. Cirrhosis patients have increased risk of complications after hip or knee arthroplasty. Acta Orthop 2015;86:108-113.

25. Poultsides LA, Ma Y, Della Valle AG, Chiu YL, Sculco TP, Memtsoudis SG. In-hospital surgical site infections after primary hip and knee arthroplasty-incidence and risk factors. $J$ Arthroplasty 2013;28:385-389.

26. Cho SW, Bhayani N, Newell P, et al. Umbilical hernia repair in patients with signs of portal hypertension: surgical outcome and predictors of mortality. Arch Surg 2012;147:864-869.

27. Cheng Y, Xiong XZ, Wu SJ, Lin YX, Cheng NS. Laparoscopic vs. open cholecystectomy for cirrhotic patients: a systematic review and meta-analysis. Hepatogastroenterology 2012;59:1727-1734.

28. Laurence JM, Tran PD, Richardson AJ, Pleass HC, Lam VW. Laparoscopic or open cholecystectomy in cirrhosis: a systematic review of outcomes and meta-analysis of randomized trials. HPB (Oxford) 2012;14:153-161.

29. Newman KL, Johnson KM, Cornia PB, Wu P, Itani K, Ioannou GN. Perioperative evaluation and management of patients with cirrhosis: risk assessment, surgical outcomes, and future directions. Clin Gastroenterol Hepatol 2020;18:2398-2414.

30. D’Amico G, Garcia-Tsao G, Pagliaro L. Natural history and prognostic indicators of survival in cirrhosis: a systematic review of 118 studies. J Hepatol 2006;44:217-231. 


\section{Supplementary material}

Supplementary Table 1 Classification of surgical procedures by ICD-9 clinical modification (CM) procedure codes*

\begin{tabular}{llr}
\hline Surgery classification & Subcategorization & ICD-9-CM procedure codes \\
\hline Cholecystectomy & & $5123,5122,5121,5124$ \\
Hernia repair & & $1711,1712,1713,1721,1722,1723,1724,4642,5300,5301,5302,5303$, \\
& & $5304,5305,5310,5311,5312,5313,5314,5315,5316,5317,5321,5329$, \\
Major abdominal & Total/partial colectomy & $5331,5339,5341,5342,5343,5349,5351,5359,5361,5362,5363,5369$ \\
& & $4572,4573,4574,4575,4576,4579,4581,4582,4583,4604,4850,4851$, \\
& Total/partial nephrectomy & $4852,4859,4862,4863$ \\
& Total/partial pancreatectomy & $554,5551,5552,5553,5554$ \\
& Total/partial splenectomy & $5251,5252,5253,5259,526,527$ \\
Major orthopedic & Total/partial gastrectomy & $4142,4143,415$ \\
& Total/partial knee, hip replacement or revision & $435,436,437,4381,4382,4389,4391,4399$ \\
& Open reduction procedures (tibia/fibula, femur, & $8151,8152,8153,8154,8155$ \\
Major cardiovascular & Coronary artery bypass graft & $7921,7922,7925,7926,7931,7932,7935,7936$ \\
& Open cardiac valve repair or replacement & $3510,3511,3512,3513,3514,3520,3521,3522,3523,3524,3525,3526$, \\
& & 3527,3528
\end{tabular}

${ }^{{ }^{*}}$ The presence of these procedure codes in any position was sufficient to classify a surgery during a hospital admission

Supplementary Table 2 National estimates and trends in surgical procedure rates per 1,000 cirrhosis hospitalizations

Discharge year Cholecystectomy Hernia repair Other major abdominal Major orthopedic Major cardiovascular Total

\begin{tabular}{|c|c|c|c|c|c|c|}
\hline 2005 & 22.1 & 10.3 & 8.9 & 9.9 & 3.3 & 54.5 \\
\hline 2006 & 19.3 & 9.6 & 8.9 & 10.1 & 3.6 & 51.4 \\
\hline 2007 & 18.2 & 10.1 & 7.9 & 10.4 & 3.0 & 49.6 \\
\hline 2008 & 18.5 & 9.7 & 7.1 & 10.9 & 2.8 & 49.1 \\
\hline 2009 & 16.0 & 9.2 & 7.2 & 10.6 & 3.6 & 46.7 \\
\hline 2010 & 16.2 & 9.7 & 6.9 & 10.2 & 2.5 & 45.5 \\
\hline 2011 & 14.7 & 8.9 & 6.5 & 10.1 & 2.8 & 43.0 \\
\hline 2012 & 13.8 & 8.7 & 6.2 & 10.5 & 2.7 & 41.9 \\
\hline 2013 & 12.4 & 9.2 & 6.3 & 10.6 & 2.9 & 41.4 \\
\hline 2014 & 12.7 & 8.5 & 6.3 & 11.4 & 3.2 & 42.0 \\
\hline Linear Trend, ( $\beta, \mathrm{P}$-value) & $\begin{array}{c}-1.004^{*} \\
(\mathrm{P}<0.001)\end{array}$ & $\begin{array}{c}-0.166^{*} \\
(\mathrm{P}=0.001)\end{array}$ & $\begin{array}{c}-0.316^{*} \\
(\mathrm{P}<0.001)\end{array}$ & $\begin{array}{c}0.083 \\
(\mathrm{P}=0.078)\end{array}$ & $\begin{array}{c}-0.052 \\
(\mathrm{P}=0.231)\end{array}$ & $\begin{array}{c}-1.454^{*} \\
(\mathrm{P}<0.001)\end{array}$ \\
\hline
\end{tabular}

